\title{
ISOMORPHISM BETWEEN AHP AND DOUBLE ENTRY BOOK KEEPING SYSTEM
}

\author{
Masaaki Shinohara* \\ Nihon University \\ Izumi-chou, Narashino \\ Chiba 275-8575, Japan \\ shinohara.masaaki@,nihon-u.ac.jp \\ Keikichi Osawa \\ Nihon University \\ Izumi-chou, Narashino \\ Chiba 275-8575, Japan \\ oosawa.keikichi@nihon-u.ac.jp
}

\begin{abstract}
An isomorphic mapping, or a one-to-one correspondence, between the Analytic Hierarchy Process and the Double Entry Book Keeping system is pointed out and the isomorphism in its detail is discussed. First, two former related studies on the suggested isomorphism, one by Professor Ludwig Mochty of Universitat Essen and the other by Professor Yuji Ijiri of Carnegie Mellon University are surveyed. The Logarithmic Analytic Hierarchy Process, which is the logarithmic least squares theory framework applied to estimating priority weights from pairwise comparison data of the Analytic Hierarchy Process, is chosen for our proposed isomorphism. The isomorphism between the AHP and the DEBK (Double Entry Book Keeping) system is summarized from four viewpoints: system topology (graph/network), what variable to choose as potential, what is skew symmetric, and the relationship between the potential and the skew symmetric matrix. It is shown that the reciprocity of pairwise comparison measurement directly contributes to the double-entry property of bookkeeping system. Through an example of the Double Entry Book Keeping system with a set of transactions during a period, the presented isomorphism is examined in detail.
\end{abstract}

Keywords: isomorphism, double entry, book keeping, logarithmic least squares, skew symmetric

\section{Introduction}

An English mathematician, Arthur Cayley, stated that the principle of double-entry book-keeping is absolutely perfect just like Euclid's theory of ratios. But Arthur Cayley didn't explain the reason for the absolute perfectness, said Yuji Ijiri in [1]. In this paper we try to explain the reason for the absolute perfectness in the principle of double-entry book-keeping by studying an isomorphism found between the Double Entry Book Keeping (DEBK) system and the Logarithmic Analytic Hierarchy Process (LAHP). The isomorphism between double-entry accounting system and AHP was first discussed in the 6th ISAHP 2001 symposium by Ludwig Mochty([2]), which will be briefly reviewed next.

\section{Former related studies by Ludwig Mochty[2] and Yuji Ijiri[1]}

Two related studies are reviewed, one by Ludwig Mochty[2] and the other by Yuji Ijiri[1].

\subsection{Isomorphism proposed by Ludwig Mochty[2]}

According to the matrix theory of double-entry accounting system, following equations (1) and (2) hold.

$$
\begin{aligned}
v(t) & =v(t-1)+S(t) \boldsymbol{I} \\
S(t) & =U(t)-U(t)^{T}
\end{aligned}
$$

Here, $v(t)$ : closing balance (column) vector for period $t$, or opening balance vector for period $t+1$, 
$S(t)$ : double-entry accounting matrix for period $t$,

$U(t)$ : transaction (general ledger) matrix for period $t$, and

$1:$ (column) vector with all the elements being 1 .

$v$ and 1 are $n \times 1$ matrixes (or column vectors), and $S$ and $U$ are $n \times n$ square matrixes, where $n$ is the number of accounts.

From the definition Eq.(2) of double-entry accounting matrix $S=\left\{s_{i j}\right\}$, skew symmetric relation of Eq.(3) holds, or matrix $\mathrm{S}$ is skew symmetric.

$$
s_{i j}=-s_{j i}
$$

For a complete-information pairwise comparison matrix $A=\left\{a_{i j}\right\}$ used in AHP, the reciprocal relation Eq.(4) is usually assumed.

$$
a_{i j}=\left(a_{j i}\right)^{-1}
$$

Taking logarithm of both sides of Eq.(4), Eq.(5) or Eq.(6) is obtained, where $\alpha_{i j}=\log a_{i j}$.

$$
\begin{gathered}
\log a_{i j}=-\log a_{j i} \\
\alpha_{i j}=-\alpha_{j i}
\end{gathered}
$$

Logarithmic pairwise comparison matrix $\log \mathrm{A}=\left\{\log a_{i j}\right\}$ is also skew symmetric.

In this sense, there is an isomorphism between double-entry accounting system and AHP.

\subsection{Isomorphism suggested by Yuji Ijiri[1]}

Ijiri insists that Cayley didn't say "Double-entry book-keeping is absolutely perfect", but Cayley said "Double-entry book-keeping is absolutely perfect just like the Euclid's theory of ratios", suggesting a formal isomorphism between a double-entry accounting matrix $S$ and a reciprocal square matrix $R$ of infinite size, called the infinite ratio matrix, given by Eq.(7).

$$
R=\left(\begin{array}{ccccc}
1 / 1 & 1 / 2 & 1 / 3 & 1 / 4 & \ldots \\
2 / 1 & 2 / 2 & 2 / 3 & 2 / 4 & \ldots \\
3 / 1 & 3 / 2 & 3 / 3 & 3 / 4 & \ldots \\
\vdots & \vdots & \vdots & \vdots & \ddots
\end{array}\right)
$$

Ijiri doesn't mention its relation with AHP, but under three conditions, any pairwise comparison matrix used in AHP can be interpreted as a square submatrix of the infinite ratio matrix $R$. The first condition is that the set of pairwise comparisons are perfectly consistent, the second condition is that priority weights are approximated to natural numbers (positive integers) and the third condition is that priority weights are all different or there exists no identical priority weight.

\section{Detailed isomorphism between Double Entry Book Keeping (DEBK) system and Logarithmic Analytic Hierarchy Process (LAHP)}

An isomorphic correspondence found between the double-entry book-keeping system and the AHP is further studied and a more detailed isomorphism is established between the double-entry book-keeping system and the logarithmic AHP, which is the logarithmic least square theory applied to estimating priority weight in AHP.

\subsection{Logarithmic AHP([3])}

The logarithmic least square theory applied to estimating priority weight is briefly reviewed from the theory of flow and potential [3]. For any pairwise comparison design graph $G(\mathrm{~V}, \mathrm{E})$, following generalized equation in an undirected manner holds from Eq.(13) of Ref.[3]. 


$$
\sum_{k \in A(i)} R_{i k} \alpha_{i k}=\sum_{k \in A(i)}\left(u_{i}-u_{k}\right) \quad i \in V
$$

Here, $\mathrm{R}_{i j}$ is the tendency of overestimation or underestimation accompanied with measurement $(i, j), \alpha_{i k}=\log a_{i k}, u_{i}=\log x_{i}\left(x_{i}:\right.$ priority weight of item $\left.i\right)$, and $A(i)=\{k \mid(i, k) \in E$ or $(k, i) \in E\}$. Considering the case of complete information of pairwise comparison measurement, letting $R_{i k}=1$, and assuming normalization condition $\Sigma u_{k}=0$, Eq.(8) is reduced to Eq.(9).

$$
\sum_{k=1}^{n} \alpha_{i k}=\sum_{k=1}^{n} u_{i}=n u_{i}
$$

Let $U_{i}=n u_{i}$, then we get Eq.(10), or Eq.(11) in matrix form.

$$
\begin{gathered}
\sum_{k=1}^{n} \alpha_{i k}=U_{i} \\
B \boldsymbol{1}=\boldsymbol{U}
\end{gathered}
$$

Here, $B=\left\{\alpha_{i k}\right\}, \boldsymbol{U}=\left\{U_{i}\right\}$, and $\boldsymbol{I}=\{1\}$.

Note that if $A=\left\{a_{i k}\right\}$ is reciprocal, then $B=\left\{\alpha_{i k}\right\}$ is skew symmetric as pointed out in Ref.[2]. Moreover, $B$ can be decomposed in such a way as $B=C-C^{T}, \mathrm{C} \geqq 0$. Here, a nonzero element of $C$ (say, $c_{i k}$ ) corresponds to an active pairwise comparison value $a_{i k}(>1)$.

\subsection{Matrix theory of double-entry book-keeping system([4])}

Let $\mathrm{S}$ be a cutset-arc incidence matrix, $T=\left\{\mathrm{t}_{i j}\right\}$ be a transaction matrix and $\boldsymbol{t}=\left\{t_{k}\right\}$ be a transaction vector for a transaction flow network $N(V, E)$. Let $n$ be the number of nodes in $V$, or the number of accounts, and $e$ be the number of arcs in $E$, or the number of positive transactions.

According to the theory of cutset analysis for the double-entry book-keeping system, proposed in Ref. [4], following equation (12) holds.

$$
f_{c}=S t
$$

Here, $f_{\mathrm{c}}$ is the transaction flow difference at cutsets. Considering a simple case, let $S$ be the node-arc incidence matrix, then Eq.(12) is reduced to Eq.(13) or Eq.(14).

$$
\begin{gathered}
\left(T-T^{T}\right) \boldsymbol{1}=S \boldsymbol{t} \\
\boldsymbol{f}=\boldsymbol{S t}
\end{gathered}
$$

Here, $\boldsymbol{f}$ is the transaction flow difference at nodes, $T$ is of $n \times n, \boldsymbol{l}$ is of $n \times 1, \mathrm{~S}$ is of $n \times e$, and t is of $e \times 1$. Any transaction matrix $T$ and transaction vector $\boldsymbol{t}$ can be made nonnegative, or $T \geqq 0$ and $\boldsymbol{t} \geqq 0$, by redefining transaction orientation, and then the spreadsheet matrix $K$ is defined by Eq.(15).

$$
K=T-T^{T}
$$

\subsection{Isomorphism between LAHP and DEBK}

Theories in preceding sections 3.1 and 3.2 are compared and are summarized as in Table 1 from four viewpoints. They are system topology (graph/network), what variable to choose as potential, what is skew symmetric, and the relationship between the potential and the skew symmetric matrix. 


\begin{tabular}{|l|l|l|}
\hline System(graph/network) & $\begin{array}{l}\text { Pairwise comparison design graph } G(V, E) \text { with } \\
\text { node set } V \text { and arc set } E . \\
\text { An item to be compared corresponds to a node and } \\
\text { a pair of compared items corresponds to an arc. }\end{array}$ & $\begin{array}{l}\text { Transaction flow network } N(V, E) \text { with node set } V \text { and } \\
\text { arc set } E . \\
\text { An account corresponds to a node and a transaction } \\
\text { which takes place between a pair of accounts } \\
\text { corresponds to an arc. }\end{array}$ \\
\hline Potential & $\begin{array}{l}\text { Logarithmic priority weight } \boldsymbol{U}=\left\{U_{i}\right\} \text {, where } U_{i}= \\
n u_{i}, u_{i}=\log x_{i}, n=\text { number of items, and } x_{i}= \\
\text { priority weight of item } i .\end{array}$ & $\begin{array}{l}\text { Transaction flow balance } \boldsymbol{f}=\left\{f_{i}\right\}, \text { where } f_{i}=\text { balance } \\
\text { at account } i .\end{array}$ \\
\hline Skew-symmetricity & $\begin{array}{l}\text { Logarithmic pairwise comparison matrix } B=\log A \\
=C-C^{T} \text {, where } C \geqq 0, A=\left\{a_{i j}\right\}, \text { and } a_{i j}= \\
\text { importance ratio of item } i \text { to item } j .\end{array}$ & $\begin{array}{l}\text { Spreadsheet matrix } K=T-T^{T}, \text { where } T \geqq 0, T=\left\{t_{i j}\right\}, \\
\text { and } t_{i j}=\text { transaction flow from account } i \text { to account } j .\end{array}$ \\
\hline $\begin{array}{l}\text { Relation between potential and } \\
\text { skew-symmetric matrix }\end{array}$ & $\begin{array}{l}\text { Relation between } \boldsymbol{U} \text { and } B \\
\boldsymbol{U}=B \mathbf{1}\end{array}$ & $\begin{array}{l}\text { Relation between } \boldsymbol{f} \text { and } K \\
\boldsymbol{f}=K \mathbf{1}\end{array}$ \\
\hline
\end{tabular}

\section{Isomorphism example}

Taking a transaction flow network shown in Fig.1 as an example, the isomorphic relation between LAHP and DEBK is explained.

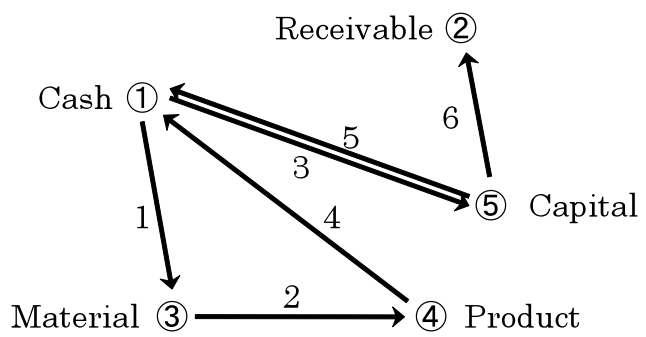

Fig.1 Transaction flow network

Transaction matrix $T$ and spreadsheet matrix $K$ are given by Eq.(16) and Eq.(17).

$$
\begin{aligned}
T & =\left(\begin{array}{ccccc}
0 & 0 & 1 & 0 & 1.1 \\
0 & 0 & 0 & 0 & 0 \\
0 & 0 & 0 & 0.8 & 0 \\
1.2 & 0 & 0 & 0 & 0 \\
0.7 & 0.5 & 0 & 0 & 0
\end{array}\right) \\
K & =\left(\begin{array}{ccccc}
0 & 0 & 1 & -1.2 & 0.4 \\
0 & 0 & 0 & 0 & -0.5 \\
-1 & 0 & 0 & 0.8 & 0 \\
1.2 & 0 & -0.8 & 0 & 0 \\
-0.4 & 0.5 & 0 & 0 & 0
\end{array}\right)
\end{aligned}
$$

Therefore, the transaction flow balance $\boldsymbol{f}$ is calculated by Eq.(18).

$$
\boldsymbol{f}=\left(T-T^{T}\right) \boldsymbol{I}=\left(\begin{array}{c}
0.2 \\
-0.5 \\
-0.2 \\
0.4 \\
0.1
\end{array}\right)
$$

Next, a pairwise comparison design graph of AHP, G(V,E), corresponding to the transaction flow network of Fig.1, N(V,E), will be studied. Since a complete set of comparison pairs are assumed, a pair of pairwise comparisons $a_{i j}=1$ and $a_{j i}=1(i \neq j)$ are assumed in the pairwise comparison matrix $A$, corresponding to a pair of non-diagonal zero elements $k_{i j}=0$ and $k_{j i}=0(i \neq j)$ in the spreadsheet matrix $K$. Then, the pairwise comparison matrix $A$ associated to the spreadsheet matrix $K$ of Eq.(17) is given by Eq.(19), where $a_{i j}=10^{k i j}$ or $K=\log A=\left\{\log _{10} a_{i j}\right\}$. 


$$
A=\left(\begin{array}{ccccc}
1 & 1 & 10 & 0.063 & 2.512 \\
1 & 1 & 1 & 1 & 0.316 \\
0.1 & 1 & 1 & 6.31 & 1 \\
15.85 & 1 & 0.16 & 1 & 1 \\
0.4 & 3.16 & 1 & 1 & 1
\end{array}\right)
$$

Since the spreadsheet matrix $K$ can be decomposed such that $K=T-T^{T}$ and $T \geqq 0, \log A=\left\{\log a_{i j}\right\}$ can be also decomposed such that $\log A=C-C^{T}$ and $C \geqq 0$. Let $C=\log _{10} D$, then the pairwise comparison matrix $D$ corresponds to the set of pairwise comparisons with their values greater than (or equal to) 1, whose design graph is shown in Fig.2, together with matrix $C$ by Eq.(20) and matrix $D$ by Eq.(21).

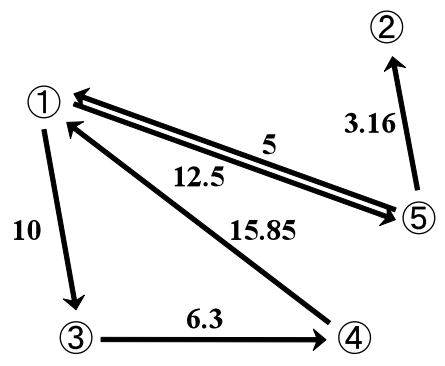

Fig.2 Pairwise comparison design graph with their pairwise comparison values graph than one

$$
\begin{aligned}
C & =\left(\begin{array}{ccccc}
0 & 0 & 1 & 0 & 1.1 \\
0 & 0 & 0 & 0 & 0 \\
0 & 0 & 0 & 0.8 & 0 \\
1.2 & 0 & 0 & 0 & 0 \\
0.7 & 0.5 & 0 & 0 & 0
\end{array}\right) \\
D & =\left(\begin{array}{ccccc}
1 & 1 & 10 & 1 & 12.5 \\
1 & 1 & 1 & 1 & 1 \\
1 & 1 & 1 & 6.3 & 1 \\
15.85 & 1 & 1 & 1 & 1 \\
5 & 3.16 & 1 & 1 & 1
\end{array}\right)
\end{aligned}
$$

Note that pairwise comparison matrix $A$ is related to $D$ by Eq.(22).

$$
A=D / D^{T} \quad\left(a_{i j}=d_{i j} / d_{j i}\right)
$$

Also note that $d_{15}=12.5$ and $d_{51}=5$ in Fig. 2 or in Eq.(21), which means that item 1 is 12.5 times better than item 5 at a measurement (or a transaction) and that item 5 is 5 times better than item 1 at another measurement (or another transaction). That is, an addition of a transaction in DEBK corresponds to an addition of a measurement in AHP. Moreover, it is interesting to notice that $c_{i j}=0$ in $\mathrm{C}$ of AHP can correspond to two cases of DEBK, a case of no transaction between $i$ and $j$, and a case of multiple transactions with their sum being 0 between $i$ and $j$. The latter case includes a case of a single transaction with its value being 0 . These two cases can be regarded almost the same in DEBK, but they cannot be regarded the same in AHP unless we employ the principle of insufficient reason.

Since this example is made such that matrix $C$ equals to matrix $T$, the priority weight potential $\boldsymbol{U}$ and the transaction flow balance $\boldsymbol{f}$ also become the same.

$$
\begin{aligned}
\boldsymbol{f} & =K \mathbf{1} \\
& =\left(T-T^{T}\right) \boldsymbol{1}
\end{aligned}
$$




$$
\begin{aligned}
& =\left(C-C^{T}\right) \boldsymbol{1} \\
& =B \boldsymbol{1} \\
& =\boldsymbol{U}
\end{aligned}
$$

$$
\boldsymbol{f}=\boldsymbol{U}=\left(\begin{array}{c}
0.2 \\
-0.5 \\
-0.2 \\
0.4 \\
0.1
\end{array}\right)
$$

Note that the transaction flow balance conservation law $\boldsymbol{1}^{T} \boldsymbol{f}=0$ holds since $\boldsymbol{1}^{T} \boldsymbol{f}=\boldsymbol{1}^{T} T \boldsymbol{1}-\boldsymbol{1}^{T} T^{T} \boldsymbol{I}=0$. Finally, the priority weights in AHP will be examined. Since $U_{i}=5 u_{i}$, the logarithmic priority weight of item $i, u_{i}\left(u_{i}=U_{i} / 5\right)$, and the priority weight of item $i, x_{i}\left(x_{i}=10^{u i}\right)$, are given by Eqs.(25) and (26).

$$
\begin{gathered}
\boldsymbol{u}=\left(\begin{array}{c}
0.04 \\
-0.1 \\
-0.04 \\
0.08 \\
0.02
\end{array}\right) \\
\boldsymbol{x}=\left(\begin{array}{c}
1.0965 \\
0.7943 \\
0.912 \\
1.202 \\
1.047
\end{array}\right)
\end{gathered}
$$

Normalizing the priority weight vector $x$ of Eq.(26) so that the sum of its elements is $1\left(x^{T} 1=1\right)$, the normalized priority weight vector is given by Eq.(27).

$$
\text { normalized } \mathbf{x}=\left(\begin{array}{l}
0.217 \\
0.157 \\
0.181 \\
0.238 \\
0.207
\end{array}\right)
$$

\section{Conclusion}

It is shown that there exists a one-to-one correspondence (or an isomorphism) between the framework of estimating priority weight vector of AHP from a pairwise comparison matrix by the logarithmic least square method and the matrix theory of double-entry book-keeping system, which is summarized in Table 1. Note that the reciprocity of pairwise comparison measurement directly contributes the double-entry property of book-keeping system. Moreover, through an example with a set of transactions during a period, the presented isomorphism is thoroughly examined and it is shown that an addition of one transaction $t_{i j}(>0)$ in the double-entry book-keeping system corresponds to an addition of one pairwise comparison measurement $a_{i j}(>1)$. That is, during a period there can exist a number of transactions cancelling and enhancing each other between two account nodes in the double-entry book-keeping system, which corresponds to a number of pairwise comparison measurements cancelling and enhancing each other between two items in the AHP.

In the theoretical development of proposed isomorphism, the complete information of pairwise comparison measurement among items and the reciprocity of pairwise comparison measurement have been assumed. Theoretical development of the isomorphism between LAHP and DEBK for the 
concept of Consistency Index and the cases of cutset analysis, incomplete information, multiple measurements, and non-reciprocal comparison matrix, are future research problems.

\section{References}

[1] Yuji Ijiri: Arthur Cayley and the Absolute Perfectness of Double-entry Bookkeeping (in Japanese), The Journal of Tokyo Keizai University, No.250, pp.33-38 (2006.3).

[2] Ludwig Mochty: The isomorphism between double-entry accounting and the analytic hierarchy process, Proceedings of ISAHP2001, pp.295-297 (2001.8).

[3] Masaaki Shinohara, Keikichi Osawa and Ken Shinohara: Flow and potential in Logarithmic Least Squares Estimation of AHP, Proceedings of ISAHP 2005, pp.12.1-12.9 (2005.7).

[4] Masaaki Shinohara and Ken Shinohara: Topological Consideration on Double-entry Bookkeeping System-Part I : Cutset analysis for transaction flow-(in Japanese), Proceeding of the $41^{\text {st }}$ Mathematical Information Research Conference, College of Industrial Technology, Nihon University, pp.1-4 (2008.12.6). 